\title{
A Methodology to Construct Approximate Constitutive Equations for Porous Rubber Elastic Materials
}

\author{
G. Perrin ${ }^{1}$ \\ 1 Institut français du pétrole, IFP, Applied Mechanics Division, 1 et 4, avenue de Bois-Préau, 92852 Rueil-Malmaison Cedex - France \\ e-mail: gilles.perrin@ifp.fr
}

\begin{abstract}
Résumé - Une méthode pour obtenir des lois de comportement approchées pour matériaux poreux à matrice caoutchoutique - Le présent article utilise des méthodes issues de la théorie de la rupture ductile, qui a été développée pour prévenir des ruptures catastrophiques de navires ou d'appareil sous pression en acier, par exemple des conduites de gaz.

De nombreuses structures sont constituées de matériaux poreux dont la matrice est incompressible et élastique, ou de matériaux de ce type qui peuvent devenir poreux en cas de sollicitations excessives. Dans le contexte de l'industrie pétrolière et gazière, c'est le cas des pièces ou structures polymères qui assurent l'étanchéité aux fluides. En effet, ces constituants, gaines étendues ou joints, sont constitués de matériaux polymères et peuvent devenir poreux en cas de décompression très rapide. La prévision de leur comportement dans de telles circonstances, et en particulier la prévision de la persistance de l'étanchéité globale, exigent de disposer d'un modèle continu qui rende compte du comportement des matériaux poreux avec une précision suffisante.

Le but du présent article est d'adapter une méthodologie issue de la théorie de la rupture ductile pour construire un tel modèle approché, par l'analyse d'une sphère creuse considérée comme Élément de Volume Représentatif (RVE) de la matière. Le problème mécanique est écrit sous une forme variationnelle, dans le cadre d'un comportement élastique du matériau constitutif de la sphère. Au voisinage du vide central, le matériau est généralement soumis à de grandes déformations. La théorie bien connue de Gurson [Gurson, A.L. (1977) J. Eng. Mater. - T. ASME, 99, 2-15] qui a été développée dans le contexte mentionné ci-dessus de la rupture ductile des aciers est adaptée pour fournir une approximation du tenseur des contraintes macroscopique (échelle du RVE). Cette approximation constitue le modèle annoncé. Les calculs sont ensuite menés explicitement pour le comportement élastique caoutchoutique néohookéen, qui est un modèle de comportement simple, représentatif de l'élasticité caoutchoutique en grandes transformations. Des calculs par la méthode des éléments finis prouvent finalement la qualité de l'approximation.
\end{abstract}

\footnotetext{
Abstract - A Methodology to Construct Approximate Constitutive Equations for Porous Rubber Elastic Materials - The present paper makes use of methods from theory of ductile fracture, which was developed to prevent catastrophic failures of steel vessels, for example gas pipelines.

A number of structures are made of porous materials, with elastic, isochoric matrix, or of such materials which may become porous when they are subjected to excessive mechanical loads. Predicting the behavior of such structures requires a continuous model which accounts well for porous materials. In the oil
} 
and gas industry context, fluid tightness sheaths or gaskets enter this category. Indeed, these constituents are often made of polymers and may become porous in case of rapid pressure drop. Forecast of their behavior in such circumstances, in particular global tightness forecast, calls for a continuous model accounting for porous materials behavior with sufficient accuracy.

The aim of the present paper is to adapt a methodology from ductile fracture theory to construct such a model, through the analysis of a hollow sphere considered as a material Representative Volume Element $(R V E)$. The mechanical problem is written under a variational form, within the framework of material elastic behavior. In the vicinity of the central void, large strains are encountered. The well-known Gurson theory [Gurson, A.L. (1977) J. Eng. Mater. - T. ASME, 99, 2-15] which was developed in the above mentioned context of steel ductile failure is adapted to provide an approximation of the macroscopic (RVE scale) stress tensor. This approximation constitutes the announced model. Computations are performed for one classical rubber elastic behavior (Neo-Hookean model), which is the simplest model combining elasticity and large strain. Finite Element Method computations show that the approximation is sufficiently tight.

\section{INTRODUCTION}

A number of materials are porous with elastic isochoric matrix (for sure, the overall material is not isochoric, because pores can expand). Some are by conception, such as foams. Also, an initially non porous material may be made porous during its lifetime: for example, cavitation can be induced by high isotropic tension, even in metals [1, $2,3]$. In a symmetric fashion, cavitation can be caused by rapid decompression of a polymer sheath or of a polymer gasket used for tightness in the context of gas or oil transportation. Indeed, in that situation, a quantity of gas is solute in the polymer matrix due to high fluid pressure, and it will return to gaseous state as pressure drops. If pressure drops more rapidly than gas can permeate out of the polymer material, gas will accumulate as bubbles inside the polymer matrix and create cavities.

The latter situation calls for a model homogenized constitutive equation for porous polymers. The present paper addresses the case when then the matrix obeys an elastic isochoric constitutive equation.

We shall admit that a hollow, thick-walled sphere constituted of an isochoric elastic matrix is an elementary volume representative of the above situation (see e.g. [4]). The inner radius of the unstrained sphere is denoted $a$ and its outer radius is equal to unity. The complete sphere volume is denoted $V_{0}$; it amounts to $\frac{4 \pi}{3}\left(1-a^{3}\right)$; the sphere outer boundary is denoted $\partial V_{0}$. Moreover, in a classical way, we qualify as "macroscopic" the data which concerns this volume globally, in average. We qualify as "microscopic" the field data: they vary as a function of position in $V_{0}$. Macroscopic and microscopic data are denoted respectively by upper and lower case letters (except for one exception: the microscopic transformation gradient, denoted $F_{i j}$ ).
The paper is organized as follows. We firstly write in Section 1 a variational expression of macroscopic internal energy, that is the global energy stored in the hollow sphere when it is subjected to some defined load. This expression cannot be transformed into any analytic form. For that reason, in Section 2, we resort to an approximation technique which provides an upper bound approximation of the macroscopic internal energy. We propose [5] to use this upper bound approximation as an elastic potential from which model constitutive equations are deduced. The formalism is then applied in Section 3 to the case of a neoHookian matrix, which is pertinent for large strain: note that large strain formalism is needed, for dilatations reach high values in the vicinity of the central void, around $\sqrt[3]{1+\Delta V_{0} / V_{0} a^{3}}$ for overall volume increase $\Delta V_{0}$. Results are finally favorably compared in Section 4 to finite element method computations of a hollow spheres of representative initial porosity subjected to uniform strain on its boundary.

\section{VARIATIONAL EXPRESSION OF MACROSCOPIC INTERNAL ENERGY}

Any homogeneous strain load can be identified through a Green-Lagrange tensor $E_{i j}$, corresponding to homogeneous macroscopic transformation $x_{i}=\Phi_{i}\left(X_{j}\right)$, where the $x_{i}{ }^{\prime} \mathrm{s}$ denote, in the transformed configuration, the co-ordinates of a material point lying at co-ordinates $X_{i}$ 's in the reference configuration. We hence have [6]:

$$
{ }^{t} \underline{\underline{\nabla \Phi}} \cdot \underline{\underline{\nabla \Phi}}=\underline{\underline{1}}+2 \underline{\underline{E}}
$$


It appears that, defining the unique square root of a symmetric tensor with positive principal values as the tensor with same principal directions and principal values changed into their respective positive square root, $\underline{\Phi}(\underline{X})$ can be written:

$$
\underline{\Phi}(\underline{X})=\sqrt{\underline{1}+2 \underline{\underline{E}}} \cdot \underline{X}
$$

up to a rigid body transform (rotation and translation). The latter transform can be omitted here, exceptionally, because it is common to the whole body under analysis (homogeneous strain condition), and can easily be accounted for globally.

On the other hand, we may define a microscopic transformation $x_{i}=\phi_{i}\left(X_{j}\right)$ as being macroscopically homogeneous if there exist some Green-Lagrange tensor $E_{i j}$ such that:

$$
\forall \underline{X} \in \partial V_{0}, \underline{\phi}(\underline{X})=\sqrt{\underline{\underline{1}}+2 \underline{\underline{E}}} \cdot \underline{X}
$$

Equation (3) must be understood as a kind of boundary conditions. It does not mean that $\phi_{i}\left(X_{j}\right)$ is homogeneous throughout $V_{0}$.

Considering the materials constituting representative volume element $V_{0}$ under study, the isochoric matrix behavior is determined through its local volumetric internal energy $\psi$ and its constant volumetric mass $\rho_{0}$. Naturally, the microscopic Piola-Kirchhoff stress tensor is then given by:

$$
\underline{\underline{\pi}}=\frac{\partial \rho_{0} \psi(\underline{\underline{e}})}{\partial \underline{\underline{e}}}
$$

where $e_{i j}$ denotes the microscopic Green-Lagrange tensor.

Now, it is obvious that the system contained in volume $V_{0}$ follows global elastic constitutive equations, since so do its elementary constituents (elastic matrix and void). It is well-known that, for homogeneous strain loads defined accordingly to boundary equations given by Equation (3), the macroscopic system $V_{0}$ obeys constitutive equation:

$$
\underline{\underline{\Pi}}=\frac{\partial \mathrm{P}_{0} \Psi(\underline{\underline{E}})}{\partial \underline{\underline{E}}}
$$

where $\Pi_{i j}$ denotes the macroscopic Piola-Kirchhoff tensor, and where volumetric macroscopic internal energy was defined as the volume average of its microscopic counterpart:

$$
\mathrm{P}_{0} \Psi(\underline{\underline{E}})=\frac{1}{V_{0}} \int_{V_{0}} \rho_{0} \psi(\underline{\underline{e}}) d \underline{X}
$$

tensor $e_{i j}$ being the microscopic Green-Lagrange strain field which is the solution of the mechanical problem: "sphere $V_{0}$ is subjected to load given by Equation (3)". In Equation (6), $\mathrm{P}_{0} \Psi$ denotes the macroscopic volumetric internal energy. At that stage, we make use of the mini- mization principle which states that the physical solution minimizes the globally strained system internal energy under two constraints:

- isochoric matrix;

- boundary conditions.

Notice that we have neglected here the possibility of multiple solutions. This seems reasonable in our objective of defining approximate constitutive equations, not establishing them in a deductive way.

Within this framework:

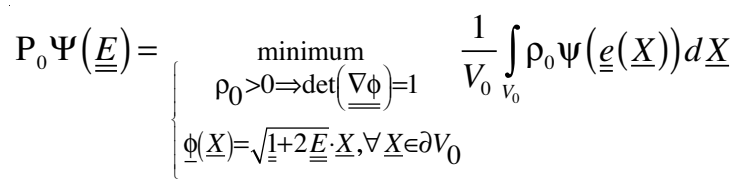

microscopic Green-Lagrange tensor being defined pointwise through:

$$
\underline{\underline{e}}=\frac{1}{2}(\underline{\underline{\nabla \phi}} \underline{\underline{\nabla \phi}}-1)
$$

In the conditions of the minimum in Equation (7), it should be clear that condition $\rho_{0}>0 \Rightarrow \operatorname{det}(\underline{\underline{\nabla \phi}})=1$ implies exactly that transformation $\phi$ be isochoric in the matrix; conversely, it can be contractant or dilatant in the void; the overall transformation hence is not necessarily isochoric.

\section{UPPER BOUND APPROXIMATION OF MACROSCOPIC INTERNAL ENERGY}

Up to this point, developments are valid for a representative volume element of any shape. Forthcoming calculation would alas not be much tractable for a general geometry.

To pursue the analysis, we hence singularize the geometry and choose the hollow sphere already presented. We then follow ideas from seminal work by Gurson [4] to construct an upper bound approximation to quantity (7): namely, we restrict the test strain fields to a very small family of microscopic transformation, which hopefully are close to the actual solution.

Practically, we choose the family of transforms parameterized by triplets $\left(\lambda_{1}, \lambda_{2}, \omega\right)$ and generated by a two-step transform:

- the isotropic volumetric increase of the central cavity of quantity $4 \pi \omega / 3$, the matrix being isochoric;

- the isochoric extension along axes $x, y$ and $z$ of respective ratios (with product unity):

$$
\frac{1}{\sqrt{\lambda_{1}}}, \frac{1}{\sqrt{\lambda_{2}}}, \sqrt{\lambda_{1} \lambda_{2}}
$$


We readily observe that these transformations are compatible with macroscopic Green-Lagrange tensors $E_{i j}$ given by equations:

$$
\begin{gathered}
E_{X X}=\frac{1}{2}\left(\frac{(\omega+1)^{2 / 3}}{\lambda_{1}}-1\right) \\
E_{Y Y}=\frac{1}{2}\left(\frac{(\omega+1)^{2 / 3}}{\lambda_{2}}-1\right) \\
E_{Z Z}=\frac{1}{2}\left((\omega+1)^{2 / 3} \lambda_{1} \lambda_{2}-1\right) \\
E_{X Y}=E_{Y Z}=E_{Z X}=0
\end{gathered}
$$

Analytic expression of microscopic transformation can easily be obtained: introducing radius $R$, co-latitude $\Theta$ and azimuth $\Phi$ in reference configuration, the above geometric description allows to write Cartesian co-ordinates in transformed configuration as:

$$
\begin{gathered}
x=\frac{1}{\sqrt{\lambda_{1}}} \sqrt[3]{\omega+R^{3}} \sin \Theta \cos \Phi \\
y=\frac{1}{\sqrt{\lambda_{2}}} \sqrt[3]{\omega+R^{3}} \sin \Theta \sin \Phi \\
z=\sqrt{\lambda_{1} \lambda_{2}} \sqrt[3]{\omega+R^{3}} \cos \Theta
\end{gathered}
$$

for $R>a$; for $R \leq a$, any regular prolongation suits. Restricting minimum (7) to this sole family, parameterized by $\lambda_{1}>0, \lambda_{2}>0$ and $\omega>-a^{3}$, provide an upper bound approximation to macroscopic potential (7):

$$
\mathrm{P}_{0} \Psi^{+}(\underline{\underline{E}})=\frac{1}{V_{0}} \int_{V_{0}} \rho_{0} \psi(\underline{\underline{e}}) d \underline{X}
$$

where Green-Lagrange strain field is deduced from transformation (14)-(16). Derivation of transform gradient $F_{i j}$ would be useless at this point. It is provided in next Section, Equations (19)-(27).

\section{APPROXIMATE CONSTITUTIVE EQUATIONS FOR POROUS ELASTIC MATERIAL (LARGE STRAIN MODEL)}

The present Section constructs the constitutive equations equivalent to potential (17), defined for a Green-Lagrange strain tensor already diagonal in framework $X, Y, Z$. The invariance of the result with respect to orientation will appear straightforward at the end of the Section.
As an example, we choose for the matrix a simple analytic isochoric isotropic elastic potential, namely NeoHookian potential [7]:

$$
\rho_{0} \psi(\underline{\underline{e}})=\mu_{0} \operatorname{tr} \underline{\underline{e}}
$$

$\mu_{0}$ being a constitutive constant.

Let us firstly calculate, at any material point in the matrix of co-ordinates $R \in[a, 1], \Theta, \Phi$, the microscopic gradient of the transformation, expressed from framework $\left(\underline{e}_{R}, \underline{e}_{\Theta}, \underline{e}_{\Phi}\right)$ in the reference geometry to framework $\left(\underline{e}_{X}, \underline{e}_{Y}, \underline{e}_{Z}\right)$ in the transformed geometry:

$$
\begin{gathered}
F_{X R}=\frac{1}{\sqrt{\lambda_{1}}}\left(\frac{\sqrt[3]{\omega+R^{3}}}{R}\right)^{-2} \sin \Theta \cos \Phi \\
F_{Y R}=\frac{1}{\sqrt{\lambda_{2}}}\left(\frac{\sqrt[3]{\omega+R^{3}}}{R}\right)^{-2} \sin \Theta \sin \Phi \\
F_{Z R}=\sqrt{\lambda_{1} \lambda_{2}}\left(\frac{\sqrt[3]{\omega+R^{3}}}{R}\right)^{-2} \cos \Theta \\
F_{X \Theta}=\frac{1}{\sqrt{\lambda_{1}}}\left(\frac{\sqrt[3]{\omega+R^{3}}}{R}\right) \cos \Theta \cos \Phi \\
F_{Y \Theta}=\frac{1}{\sqrt{\lambda_{2}}}\left(\frac{\sqrt[3]{\omega+R^{3}}}{R}\right) \cos \Theta \sin \Phi \\
F_{Y \Phi}=\frac{1}{\sqrt{\lambda_{2}}}\left(\frac{\sqrt[3]{\omega+R^{3}}}{R}\right) \cos \Phi \\
F_{X \Phi}=-\sqrt{\lambda_{1} \lambda_{2}}\left(\frac{1}{\sqrt{\lambda_{1}}}\left(\frac{\sqrt[3]{\omega+R^{3}}}{R}\right) \sin \Theta\right. \\
F_{Z \Phi}=0 \\
\sin \Phi
\end{gathered}
$$

from which we deduce the microscopic Green-Lagrange tensor $\underline{\underline{e}}=\left({ }^{t} \underline{\underline{F}} \underline{\underline{F}}-1\right) / 2$, and the volumetric microscopic internal energy corresponding to Equations (14)-(16): 


$$
\begin{aligned}
& \mu_{0} \operatorname{tre}=\frac{\mu_{0}}{2}\left[-3+\left(\frac{\sqrt[3]{\omega+R^{3}}}{R}\right)^{-4} \times\right. \\
& \left(\sin ^{2} \Theta\left(\frac{\cos ^{2} \Phi}{\lambda_{1}}+\frac{\sin ^{2} \Phi}{\lambda_{2}}\right)+\lambda_{1} \lambda_{2} \cos ^{2} \Theta\right)+ \\
& \left(\frac{\sqrt[3]{\omega+R^{3}}}{R}\right)^{2}\left(\frac{\cos ^{2} \Theta \cos ^{2} \Phi+\sin ^{2} \Phi}{\lambda_{1}}+\right. \\
& \left.\left.\frac{\cos ^{2} \Theta \sin ^{2} \Phi+\cos ^{2} \Phi}{\lambda_{2}}+\lambda_{1} \lambda_{2} \sin ^{2} \Theta\right)\right]
\end{aligned}
$$

Secondly, we average the latter quantity on the sphere $S_{r}$ of radius $r$ in the transformed geometry to get:

$$
\begin{aligned}
\left\langle\mu_{0} \operatorname{tre} \underline{\underline{e}}\right\rangle_{S_{r}} & =-\frac{3 \mu_{0}}{2}+\frac{\mu_{0}}{6}\left\{2\left(\frac{\sqrt[3]{\omega+R^{3}}}{R}\right)^{2}+\left(\frac{\sqrt[3]{\omega+R^{3}}}{R}\right)^{-4}\right\} \\
& \times\left(\frac{1}{\lambda_{1}}+\frac{1}{\lambda_{2}}+\lambda_{1} \lambda_{2}\right)
\end{aligned}
$$

Averaging Equation (29) on $r \in[0,1]$ yields an expression for the macroscopic volumetric internal energy (17); it appears that variables are partially separated:

$$
\mathrm{P}_{0} \Psi^{+}=-\frac{3 \mu_{0}}{2}\left(1-a^{3}\right)+\frac{\mu_{0}}{6} f\left(\lambda_{1}, \lambda_{2}\right) g\left(\omega, a^{3}\right)
$$

where:

$$
\begin{gathered}
f\left(\lambda_{1}, \lambda_{2}\right)=\frac{1}{\lambda_{1}}+\frac{1}{\lambda_{2}}+\lambda_{1} \lambda_{2} \\
g\left(\omega, a^{3}\right)=2 g_{2}\left(\omega, a^{3}\right)+g_{-4}\left(\omega, a^{3}\right) \\
\text { with } g_{n}\left(\omega, a^{3}\right)=\int_{a^{3}}^{1}\left(\frac{\omega+u}{u}\right)^{\frac{n}{3}} d u, \quad \forall n \in Z
\end{gathered}
$$

Use of notations (31)-(32) is justified by the forthcoming necessity of deriving the $g_{n}$ 's with respect to variable $\omega$, which results, using integration by parts, in, $\forall n \in Z$ :

$$
\frac{\partial g_{n}\left(\omega, a^{3}\right)}{\partial \omega}=\frac{1}{\omega}\left[g_{n}\left(\omega, a^{3}\right)+a^{3}\left(1+\frac{\omega}{a^{3}}\right)^{\frac{n}{3}}-(1+\omega)^{\frac{n}{3}}\right]
$$

Isotropy of overall energy is no more apparent. It will be clear again in the following, when constitutive equations are presented in terms of a strain-stress relationship (Equations 41-47).

The following of the present Section is devoted to obtaining the macroscopic constitutive equation, as an expression of the macroscopic Piola-Kirchhoff principal extra-stresses $\Pi_{X X}, \Pi_{Y Y}, \Pi_{Z Z}$ as functions of the principal values of the macroscopic Green-Langrange tensor $E_{X X}$, $E_{Y Y}, E_{Z Z}$. We shall make use of differentiation formula (no summation over repeated index $K$ ):
$\Pi_{K K}=\frac{\partial \mathrm{P}_{0} \Psi^{+}}{\partial E_{K K}}=\frac{\partial \mathrm{P}_{0} \Psi^{+}}{\partial \lambda_{1}} \frac{\partial \lambda_{1}}{\partial E_{K K}}+\frac{\partial \mathrm{P}_{0} \Psi^{+}}{\partial \lambda_{2}} \frac{\partial \lambda_{2}}{\partial E_{K K}}+\frac{\partial \mathrm{P}_{0} \Psi^{+}}{\partial \omega} \frac{\partial \omega}{\partial E_{K K}}$

To this aim, we need to obtain expressions for factors $\frac{\partial \lambda_{1}}{\partial E_{K K}}$ and similar. This can be done as follows. Firstly, inversion of system (10)-(13) yields:

$$
\left\{\begin{array}{l}
\lambda_{1}=\left(\frac{\left(1+2 E_{Y Y}\right)\left(1+2 E_{Z Z}\right)}{\left(1+2 E_{X X}\right)^{2}}\right)^{1 / 3} \\
\lambda_{2}=\left(\frac{\left(1+2 E_{X X}\right)\left(1+2 E_{Z Z}\right)}{\left(1+2 E_{Y Y}\right)^{2}}\right)^{1 / 3} \\
1+\omega=\sqrt{\left(1+2 E_{X X}\right)\left(1+2 E_{Y Y}\right)\left(1+2 E_{Z Z}\right)}
\end{array}\right.
$$

whence:

$$
\left\{\begin{array}{l}
\lambda_{1}=\left(1+2 E_{X X}\right)^{-\frac{2}{3}}\left(1+2 E_{Y Y}\right)^{\frac{1}{3}}\left(1+2 E_{Z Z}\right)^{\frac{1}{3}} \\
\lambda_{2}=\left(1+2 E_{X X}\right)^{\frac{1}{3}}\left(1+2 E_{Y Y}\right)^{-\frac{2}{3}}\left(1+2 E_{Z Z}\right)^{\frac{1}{3}} \\
1+\omega=\left(1+2 E_{X X}\right)^{\frac{1}{2}}\left(1+2 E_{Y Y}\right)^{\frac{1}{2}}\left(1+2 E_{Z Z}\right)^{\frac{1}{2}}
\end{array}\right.
$$

Let us introduce the principal value of the Cauchy tensor $C_{K K}=1+2 E_{K K}$. Differentiation of Equation (36) hence leads to:

$$
\left(\begin{array}{lll}
\frac{\partial \lambda_{1}}{\partial E_{X X}} & \frac{\partial \lambda_{1}}{\partial E_{Y Y}} & \frac{\partial \lambda_{1}}{\partial E_{Z Z}} \\
\frac{\partial \lambda_{2}}{\partial E_{X X}} & \frac{\partial \lambda_{2}}{\partial E_{Y Y}} & \frac{\partial \lambda_{2}}{\partial E_{Z Z}} \\
\frac{\partial \omega}{\partial E_{X X}} & \frac{\partial \omega}{\partial E_{Y Y}} & \frac{\partial \omega}{\partial E_{Z Z}}
\end{array}\right)=\frac{1}{3}\left(\begin{array}{ccc}
-4 \frac{\lambda_{1}}{C_{x x}} & 2 \frac{\lambda_{1}}{C_{Y Y}} & 2 \frac{\lambda_{1}}{C_{Z Z}} \\
2 \frac{\lambda_{2}}{C_{x x}} & -4 \frac{\lambda_{2}}{C_{Y Y}} & 2 \frac{\lambda_{2}}{C_{Z Z}} \\
3 \frac{1+\omega}{C_{x x}} & 3 \frac{1+\omega}{C_{Y Y}} & 3 \frac{1+\omega}{C_{Z Z}}
\end{array}\right)
$$

and the awaited expression for the Piola-Kirchhoff stress tensor non-zero components is readily obtained:

$$
\left\{\begin{aligned}
& \Pi_{X X}=\frac{\mu_{0}}{6 C_{X X}}[ \frac{2}{3}\left(\frac{2}{\lambda_{1}}-\frac{1}{\lambda_{2}}-\lambda_{1} \lambda_{2}\right) g\left(\omega, a^{3}\right)+ \\
&\left.\left(\frac{1}{\lambda_{1}}+\frac{1}{\lambda_{2}}+\lambda_{1} \lambda_{2}\right)(1+\omega) g_{, \omega}\left(\omega, a^{3}\right)\right] \\
& \Pi_{Y Y}=\frac{\mu_{0}}{6 C_{Y Y}}\left[\frac{2}{3}\left(-\frac{1}{\lambda_{1}}+\frac{2}{\lambda_{2}}-\lambda_{1} \lambda_{2}\right) g\left(\omega, a^{3}\right)+\right. \\
&\left.\left(\frac{1}{\lambda_{1}}+\frac{1}{\lambda_{2}}+\lambda_{1} \lambda_{2}\right)(1+\omega) g_{, \omega}\left(\omega, a^{3}\right)\right] \\
& \Pi_{Z Z}=\frac{\mu_{0}}{6 C_{Z Z}}\left[\frac{2}{3}\left(-\frac{1}{\lambda_{1}}-\frac{1}{\lambda_{2}}+2 \lambda_{1} \lambda_{2}\right) g\left(\omega, a^{3}\right)+\right.\left.\left(\frac{1}{\lambda_{1}}+\frac{1}{\lambda_{2}}+\lambda_{1} \lambda_{2}\right)(1+\omega) g_{, \omega}\left(\omega, a^{3}\right)\right]
\end{aligned}\right.
$$


To obtain the macroscopic Cauchy stress tensor, we use the usual transport relationship:

$$
\underline{\underline{\Sigma}}=\frac{\mathrm{P}}{\mathrm{P}_{0}} \underline{\underline{F}} \underline{\underline{\Pi}}^{t} \underline{\underline{F}}
$$

that is, for any $K$ :

$$
\Sigma_{K K}=\frac{C_{K K}}{1+\omega} \Pi_{K K}
$$

The Cauchy stress tensor naturally writes down to a sum of its deviator $\underline{\underline{S}}$ and its hydrostatic scalar $\Sigma_{m}$ :

$$
\underline{\underline{\Sigma}}=\underline{\underline{S}}+\Sigma_{m} \underline{\underline{1}}
$$

with:

$$
\begin{gathered}
\underline{\underline{S}}=\frac{\mu_{0}}{9} g\left(\omega, a^{3}\right) \underline{\underline{T}} \\
T_{X X}=\frac{2}{\lambda_{1}}-\frac{1}{\lambda_{2}}-\lambda_{1} \lambda_{2} \\
T_{Y Y}=-\frac{1}{\lambda_{1}}+\frac{2}{\lambda_{2}}-\lambda_{1} \lambda_{2} \\
T_{Z Z}=-\frac{1}{\lambda_{1}}-\frac{1}{\lambda_{2}}+2 \lambda_{1} \lambda_{2} \\
\Sigma_{m}=\frac{\mu_{0}}{6} g_{, \omega}\left(\omega, a^{3}\right)\left(\frac{1}{\lambda_{1}}+\frac{1}{\lambda_{2}}+\lambda_{1} \lambda_{2}\right)
\end{gathered}
$$

Non diagonal $\underline{\underline{T}}$ component are null. In order to make apparent the problem symmetries, notice that $\underline{\underline{T}}$ can be written in tensor form as:

$$
\underline{\underline{T}}=\underline{\underline{U}}-\left(\frac{1}{3} \operatorname{tr} \underline{\underline{U}}\right) \underline{\underline{1}} \quad \text { with } \underline{\underline{U}}=\frac{3 \underline{\underline{F}} \cdot{ }^{t} \underline{\underline{\underline{F}}}}{\sqrt[3]{\operatorname{det} \underline{\underline{C}}}}
$$

(for diagonal $\underline{\underline{F}}$, as considered here, notice that $\underline{\underline{F}} \cdot{ }^{t} \underline{\underline{F}}=\underline{\underline{C}}$ ).

Set of analytic Equations $\{(31)-(33),(36),(41)-(46)\}$ define model macroscopic constitutive equations for a isochoric elastic hollow sphere element. We note that integrals $g_{2}$ and $g_{-4}$ do not admit any expression in terms of usual functions, even if $g_{2}$ is identical to hypergeometric function $H([1 / 3,1 / 3],[4 / 3], u / \omega)$. This is not a difficulty in practice for, once the material is chosen, initial porosity is known and the geometric parameter $a$ is fixed. Then both integrals reduce to a function of one variable, which can easily be tabulated before starting any structural computation.

\section{TESTING THE MODEL AGAINST FEM CELL COMPUTATIONS}

Model \{(31)-(33), (36), (41)-(46)\} was obtained, in the particular case of a Neo-Hookian matrix, through approaching exact potential (6) by Equation (17). The hopefully judicious choice of the family of transformations (14)-(16) is believed to lead to a good approximation, by analogy with the excellent result of Gurson [4] for a ideal rigid plastic matrix. It is natural, however, to test in some way the accuracy of this approximation.

One way to test it is to perform numerical computations of the hollow sphere cell by means of the Finite Element Method (FEM). This is known to provide quite an accurate approximation of Equation (6). For that reason, we compare the model to some FEM computations.

FEM computations were realized [8] for a hollow sphere of internal radius $a=0.25$, corresponding to initial void volume fraction $1.5625 \%$. The matrix follows NeoHookian behavior (18) with $\mu_{0}=1$. We consider axially symmetric loads, with $\lambda=\lambda_{1}=\lambda_{2}$. Loads corresponding to couples $(\lambda, \omega)$ given in Table I were considered. Corresponding values of $E_{\mathrm{XX}}$ and $E_{\mathrm{ZZ}}$ are computed accordingly to:

$$
\left\{\begin{array}{l}
\lambda=\left(\frac{1+2 E_{Z Z}}{1+2 E_{X X}}\right)^{1 / 3} \\
1+\omega=\left(1+2 E_{X X}\right) \sqrt{1+2 E_{Z Z}}
\end{array}\right.
$$

Because of axial symmetry, Cauchy stresses derived from the model amount to:

$$
\left\{\begin{array}{l}
\Sigma_{X X}=\Sigma_{Y Y}=\Sigma_{m}-\frac{1}{2} S_{Z Z} \\
\Sigma_{Z Z}=\Sigma_{m}+S_{Z Z}
\end{array}\right.
$$

with:

$$
\begin{aligned}
& S_{Z Z}=\frac{2 \mu_{0}}{9} g\left(\omega, a^{3}\right)\left(-\frac{1}{\lambda}+\lambda^{2}\right) \\
& \Sigma_{m}=\frac{\mu_{0}}{6}\left(\frac{2}{\lambda}+\lambda^{2}\right) g_{\text {, }}\left(\omega, a^{3}\right)
\end{aligned}
$$

Considering FEM computations, macroscopic Cauchy stresses $\Sigma_{X X}^{M E F}$ and $\Sigma_{Z Z}^{M E F}$ are obtained through averaging microscopic Cauchy stresses on volume $V_{0}$ [8].

Table 1 provides comparison results for two different loads. 
TABLE 1

Cauchy stresses obtained by model $\{(31)-(33),(36),(41)-(46)\}$ compared to Cauchy stresses obtained through MEF computations, and relative error

\begin{tabular}{c|c|c|c}
\hline Loads & $\begin{array}{c}\text { Stresses from } \\
\text { model }\end{array}$ & $\begin{array}{c}\text { Stresses from } \\
\text { MEF computation }\end{array}$ & Error \\
\hline$\lambda=1.01$ & $\Sigma_{X X}=0.0309$ & $\Sigma_{X X}^{M E F}=0.0313$ & $1 \%$ \\
$\omega=0.0005$ & $\Sigma_{Z Z}=0.0605$ & $\Sigma_{Z Z}^{M E F}=0.0605$ & $<1 \%$ \\
$\mathrm{E}_{X X}=-0.479 \%$ & & & \\
$\mathrm{E}_{Z Z}=1.022 \%$ & & & \\
\hline$\lambda=1.1$ & $\Sigma_{X X}=0.438$ & $\Sigma_{X X}^{M E F}=0.446$ & $2 \%$ \\
$\omega=0.01$ & $\Sigma_{Z Z}=0.735$ & $\Sigma_{Z Z}^{M E F}=0.745$ & $1 \%$ \\
$E_{X X}=-4.24 \%$ & & & \\
$E_{Z Z}=10.9 \%$ & & & \\
\hline
\end{tabular}

\section{CONCLUSION}

The approximate model was derived using a loading which seems very special: a hollow sphere geometry. The reasons to choose this heuristic are the following:

- it is the simplest geometry to account for a porous material;

- loads chosen constitute the easiest way to couple isotropic dilatation and deviatoric strains. Because the methodology is not deductive, quality of the approximation has to be tested.

The present paper shows it is quite precise for volume strain up to $1 \%$ and deviatoric strain up to $10 \%$.

It would be interesting to check the quality of the approximation also for larger overall strain. But this needs amelioration of the FEM computation technique (remember that that $1 \%$ isotropic stretching of a $1 \%$ voided sphere leads to a maximal deviatoric stretch approaching $25 \%$ in the vicinity of the central void) and it is left for future work.

Generalization to an elastic isochoric matrix obeying an elastic potential which can be expressed as a polynomial function of the invariants of the Green-Lagrange tensor (Mooney-Rivlin potential, see [7], is a simple example) will lead, after possibly fastidious calculations, to a macroscopic potential which can be written as a sum of a few terms of the form:

$$
g_{q}\left(\omega, a^{3}\right) h\left(\lambda_{1}, \lambda_{2}\right)
$$

for different $q$ 's. On the other hand, choice of a microscopic elastic potential which is expressed as a function of the dilatations (for example potentials of Varga or Ogden, see [6]) lead to more severe difficulties. The reason is that extraction of dilatation from the Cauchy tensor issued from Equations (18)-(27) provides an expression with radicals, the average of which over volume $V_{0}$ does not come down to a sum of partially separated variables as Equation (49). These issues also are left for future work.

\section{ACKNOWLEDGMENTS}

The author acknowledges Laurent Cangémi for some computational results and Jean-Baptiste Leblond for useful comments.

\section{REFERENCES}

1 Mc Clintock, F.A. (1968) A criterion for double fracture by the growth of holes, J. Appl. Mech., 35, 363.

2 Rice, J.R. and Tracey, D.M. (1969) On the Ductile Enlargement of Voids in Triaxiol Stress Fields, J. Mech. Phys. Solid, 17, 201-217.

3 Morini, B., Mudry, F. and Pineau, A. (1985) Experimental Study of Cavity Growth in Ductile Rupture, Eng. Fract. Mech., 22, 989 .

4 Gurson, A.L. (1977) Continuum Theory of Ductile Rupture by Void Nucleation and Growth: Part I - Yield Criteria and Flow Rules for Porous Ductile Media, J. Eng. Mater. - T. ASME, 99, 2-15.

5 Perrin, G. (2003) Quelques contributions à la mécanique de la rupture et de l'endommagement pour les industries pétrolière et parapétrolière, Mémoire d'habilitation à diriger des recherches, Université Pierre et Marie Curie, Paris.

6 Salençon, J. (1996) Mécanique des milieux continus, cours à l’École polytechnique, Ellipses Ed., Paris.

7 Green, A.E. and Zerna, W. (1968) Theoretical elasticity, Oxford University Press, Londo.

8 Cangémi, L. (2002) Private communication, IFP.

Final manuscript received in December 2005 